\title{
Protée
}

\section{L’Art de concocter des titres en cuisine}

\section{Jean-Jacques Boutaud}

Volume 36, numéro 3, hiver 2008

Le titre des œuvres : accessoire, complément ou supplément

URI : https://id.erudit.org/iderudit/019631ar

DOI : https://doi.org/10.7202/019631ar

Aller au sommaire du numéro

Éditeur(s)

Département des arts et lettres - Université du Québec à Chicoutimi

ISSN

0300-3523 (imprimé)

1708-2307 (numérique)

Découvrir la revue

Citer cet article

Boutaud, J.-J. (2008). L’Art de concocter des titres en cuisine. Protée, 36(3), 23-33. https://doi.org/10.7202/019631ar

\section{Résumé de l'article}

Les titres marquent l'histoire culinaire, mais ils agissent très longtemps en trompe-l'oeil. Ils annoncent cuisine et recettes, mais s'évadent aussi vite dans l'encyclopédisme du goût et de la table, tant saveur et savoir font bon ménage. Inlassablement, à travers ouvrages et revues, ils " attaquent » la nourriture sous tous les angles, au point d'offrir, dans leur ensemble, un vaste espace figuratif du goût, articulé à de nombreux niveaux. Derrière le titre, se dessine très longtemps le discours de la compétence et de l'expertise. Mais avec les ressources d'Internet et la prolifération des blogues, la fonction poétique des titres se conjugue à une nouvelle éthique de la cuisine. 


\section{L'ART DE CONCOCTER DES TITRES EN CUISINE}

JEAN-JACQUES BOUTAUD

À partir du XIVe siècle, apparaissent les premiers livres de cuisine rédigés depuis les lointaines références à Apicius (Antiquité). Ils inaugurent un genre à la fois bien stabilisé autour des activités de préparation alimentaire et, dans le même temps, attiré par tout ce qui touche aux aliments, au goût, à la table. Entre saveur et savoir (qui ont la même étymologie: sapere), sagesse et plaisir, savoir-faire (cuisine, recettes) et faire savoir (origine, histoire, bonnes manières), il est difficile de se limiter à la physique des aliments ou à la pratique des recettes, quand le goût et la table en appellent aux valeurs tout autant qu'aux saveurs et quand la cuisine s'érige en art, en morale, en science et en culture.

Dans la profusion des titres qui alimentent, depuis des siècles, la littérature culinaire et gastronomique, les références sont innombrables ou constituent, au mieux, un corpus ouvert, d'une très grande diversité thématique. La France, à elle seule, dotée du plus grand corpus culinaire dans le monde - peut-être avec la Chine - a toujours su enrichir son histoire et sa créativité culinaires d'une littérature foisonnante. Mais quelques ouvrages font date et marquent des étapes particulièrement importantes de cette histoire culinaire, associée à des époques, des noms, des styles, des spécialités.

Dans sa première édition de 1938, le Larousse gastronomique propose «une bibliographie culinaire et gastronomique, ancienne et moderne», où figurent près d'une centaine d'auteurs et 140 titres d'ouvrages - un corpus déjà très riche en informations. Dans son apparente unité, il laisse apparaître une grande diversification des écrits et un éclatement des genres dans la catégorie générique du culinaire et du gastronomique. Il est question, ça et là, de s'exprimer sous différentes formes: traité, guide, dictionnaire, livre, almanach, memorandum, manuel, etc. En réalité, les formes énonciatives prennent souvent des libertés par rapport au mode discursif annoncé. La délimitation des genres s'en trouve brouillée, pourrait-on dire, mais davantage assouplie. On recourt souvent pour illustration à la Physiologie du goût de Brillat-Savarin (1825) qui a l'ambition d'un traité scientifique mais qui prend, d'entrée de jeu, un tour poétique, un caractère moral, une portée philosophique. Et que dire du Grand Dictionnaire de cuisine d'Alexandre Dumas, destiné à «être lu par les gens du monde et pratiqué par les 
gens de l'art» (1873: 104). Cela nous engage, ici, non pas à une discussion littéraire sur le genre, mais bien à traiter l'ensemble des titres, proposés dans ce corpus, comme les occurrences d'un système qui, d'une part, renvoie aux stratégies discursives assumées par le titre, pour définir l'angle d'approche à donner à la question culinaire et gastronomique - et peut-être mieux en jouer -, et, d'autre part, déploie une axiologie autour de ce que l'on peut définir comme un système de valeurs associées aux aliments, à la cuisine, au goût. Dans la dispersion et la profusion des titres, il s'agira donc de retrouver un principe organisateur et des logiques signifiantes qui éclaireront des dimensions sémiotiques du monde culinaire.

Notre hypothèse est que des logiques de sens sont à l'œuvre dans le marché inépuisable de l'édition culinaire et gastronomique, dont les titres composent avec les modalités pratiques du livre de recettes et les modalités mythiques du livre d'art (mythologie du chef, de terroir, du produit, etc.). L'histoire culinaire s'est longtemps confondue avec un surmoi de connaissance, d'expertise, selon une logique de savoir rapportée à une figure, à un art, à un talent ou, pour le moins, à une technique, mais pas seulement à une pratique. Avec l'apparition d'Internet et des blogues, le mouvement s'inverse: un rapport de proximité et de familiarité s'installe dans une relation avant tout ludique et pratique avec la cuisine. À charge, pour le titre, de préfigurer cet ethos. À charge aussi, pour nous, de comprendre comment s'est développé et déplacé cet horizon imaginaire, depuis les premiers ouvrages de référence jusqu'aux titres les plus inventifs, dans la liberté créative d'Internet.

Mais avant de suivre cette trajectoire du sens, quelques observations s'imposent.

\section{REMARQUES LIMINAIRES}

La démarche choisie ne répond pas à des critères quantitatifs voulant qu'on s'appuie, par exemple, sur des inventaires, des répertoires, des rubriques de toutes sortes (vins, aliments, cuisine, pays, auteur, etc.), qui, sans espoir d'exhaustivité, donneraient malgré tout le sentiment, avec des corpus aussi étendus que possible, de réunir un maximum de données pour étudier les titres en gastronomie. Même s'il faut avoir les ressources de toutes ces banques de données ou de références (historiques, bibliographiques, lexicographiques, etc.), la question du titre se pose néanmoins en termes qualitatifs dès lors qu'on cherche à comprendre la position discursive qu'il manifeste (discours savant, esthétique, pratique, etc.) et les logiques de signification qui se créent à l'intérieur de ce que l'on pourrait définir comme un système de production du discours culinaire. Le titre s'inscrit alors dans un genre, mais il peut aussi se situer dans une logique de programme, de contrat, en assurant différentes fonctions: didactique, poétique, ludique, d'accroche, etc.

La deuxième observation concerne l'objet même de ce discours, ce sur quoi le titre se recentre, ce autour de quoi il gravite: fait-il référence aux aliments, aux chefs, à la table, aux saveurs, aux petits plats ou à la grande cuisine? Là encore, il ne s'agit pas de couvrir tout le plan de projection du monde alimentaire: «La cuisine est un perfectionnement de l'alimentation.

La gastronomie est un perfectionnement de la cuisine elle-même» (Revel, 1979: 32).

Les sphères du culinaire et du gastronomique créent donc un premier recentrage par rapport à toutes les références disponibles sur la nourriture (en matière de plaisir ou de santé, par exemple), les aliments (origine, histoire, propriétés) et l'alimentation au sens large.

Cependant, les termes "aliments", "cuisine», «goût» et "gastronomie» jouent de leur contiguïté, de leur relation métonymique. Cela rend difficile la délimitation d'un périmètre pour l'étude des titres, mais contribue, dans le même temps, à dessiner, audelà d'un objet, un territoire, celui de l'imaginaire alimentaire: côté cuisine ou côté table; côté recettes ou côté saveur; entre ethos, inscription dans un monde ou un mode alimentaire, et praxis, possibilité de faire, d'agir, de se réaliser à l'intérieur de ce monde. D'un point à l'autre, d'un titre à l'autre, c'est tout l'univers du goût alimentaire qui se recompose, avec ses figures avant tout actorielles (personnalisation dans le titre), 
actantielles (sélection, dans le titre, d'un élément particulier du goût) et thématiques (titre indexé à un lieu, une époque, une pratique).

La troisième observation n'est pas la moins embarrassante, quand, pour analyser les titres culinaires, on se place non pas, en priorité, sur un terrain linguistique et lexicographique, mais dans une perspective avant tout redevable à la sémiotique et à la communication. À quoi s'applique en propre la notion de titre dans le monde culinaire? Aux ouvrages et aux revues, certes. On pourrait penser aussi aux recettes. Ainsi, sur Internet, LaMarmite. com propose une recherche par "titre», mais l'usage le plus fréquent est de renvoyer au «nom» d'une recette. En conséquence, on ne s'attardera pas à ce domaine d'application, encore mal stabilisé par rapport à l'utilisation du mot "titre». Il en est de même pour les menus et les plats, plus volontiers rattachés à des noms qu'à des titres. En revanche, Internet, avec les sites et les blogues culinaires, offre aujourd'hui un large terrain d'exploration pour analyser les titres et les logiques de signification qui peuvent ressortir de leur explosion créative. C'est pourquoi nous y ferons référence, mais dans un registre nouveau par rapport aux titres des ouvrages et des revues culinaires, car ils jouent, évidemment, des propriétés de leur support et changent, considérablement, la logique titulaire.

En première lecture, on note donc un déplacement progressif du titre, au fil du temps et des supports: lié initialement à la science (recettes et dictionnaires), puis aux arts culinaires (revues), avant de laisser place à l'expérience (Internet). Mais l'évolution dans les titres n'est pas aussi tranchée d'une époque à l'autre ou d'un support à l'autre. Il faudra saisir les nuances qui donnent aux titres leur saveur et dessinent des contours de sens moins tranchés: d'abord voir, à travers les grands ouvrages de l'histoire culinaire, comment le titre préfigure un genre, de façon transparente ou trompeuse; puis recomposer l'assiette sémantique, ou l'espace figuratif du goût, à partir des titres des ouvrages et revues; enfin, saisir comment le réseau Internet et les blogues culinaires annoncent, dans la poétique même du titre, une éthique de vie.
Dans le déploiement de l'espace figuratif, tel qu'il est décrit par Fontanille (2007) et peut s'appliquer, pour nous, à l'imaginaire culinaire, la forme de vie constitue un niveau de pertinence sémiotique qui complète les autres niveaux de manifestation: les signes sensoriels (formes, couleurs, etc.), les textes (recettes, par exemple), les objets (aliments et cuisine), les scènes prédicatives (le faire culinaire), les stratégies globales (dimension du plaisir, de la santé, du lien, etc.) et, donc, les formes de vie (associées aux valeurs, à l'ethos). Autrement dit, on observera une figurativité du goût, des signes alimentaires les plus discrets aux choix de vie rendus expressifs dans notre rapport aux aliments, à la cuisine, à la table. Les titres vont jouer de tous ces rapports.

\section{TITRES SAVANTS - TITRES SAVOUREUX}

Nos références sont limitées aux éditions françaises qui ont eu tant d'influence sur l'histoire culinaire. Certes, on peut toujours imaginer une extension du champ d'étude, d'un continent à l'autre, d'une période à l'autre. Pensons à la Chine, avec plus de 4000 ans d'histoire culinaire et une tradition aussi longue d'écrivains et de poètes qui cultivent l'art d'être gourmet et l'expérience esthétique des aliments. On n'ose toutefois pas imaginer où cela entraînerait le fil de cette étude, ou, plutôt, on peut faire l'hypothèse que cela ne modifierait pas vraiment ce qui nous occupe, à savoir, à ce stade, la recherche d'un premier système de positions discursives manifestées dans les titres, à l'égard du monde culinaire.

À parcourir la centaine de titres dans le Larousse gastronomique - pour l'histoire culinaire entre Le Viandier (1370, date incertaine), le grand document synthétisant la cuisine médiévale, et Le Guide culinaire d'Escoffier, aide-mémoire de cuisine pratique (1903), bible de tous les cuisiniers professionnels -, on observe une grande variété de genres. Bien qu'ils ressortissent globalement à ce que l'on désigne comme des traités de cuisine ${ }^{1}$, ils renvoient, en apparence, à des genres pour le moins marqués par la diversité: par exemple, l'Almanach des gourmands de Grimod de la Reynière (1803 à 1810) ou son Manuel des amphitryons (1808); 
les dictionnaires comme le Nouveau Dictionnaire de cuisine, d'office et de pâtisserie de Borel (1825), le Dictionnaire des aliments et boissons d'Aulagnier (1830) ou le Grand Dictionnaire de cuisine de Dumas (1873); les Livres de cuisine appliqués par Gouffé $(1867,1869)$ à tous les registres; les traités, ceux qui se revendiquent comme tels, à l'image du Traité de l'office d'Etienne (1845). Annoncés sous forme de lettres, les Lettres gourmandes de Monselet (1877), de guides, Le Guide culinaire d'Escoffier (1903), ou d'abécédaire, l'Alphabet de la Ménagère de Driessens (1894); tous les genres trouvent leur expression.

La pratique culinaire ou ses agents passent par tous les qualificatifs: méthodique (La Varenne, 1662), moderne (La Chapelle, 1835), bourgeoise (Menon, 1746), classique (Dubois et Bernard, 1856), artistique (Dubois, 1872), etc. On pourrait prolonger à l'envi la liste des qualificatifs qui, dès le titre, affichent ou présentent la cuisine sous une dimension privilégiée: le temps, l'espace, un secteur appliqué, un créateur, un style, etc.

Les références à la cuisine forment ainsi, à elles seules, un petit système. Sur l'axe temporel, se situe tout ce qui renvoie à l'ancien et au moderne, au classique et à l'actuel (cuisine du jour, d'aujourd'hui). Mais, paradoxalement, pendant des siècles de littérature culinaire, on fait peu référence au temps de cuisson et aux proportions, soit parce qu'une forme de savoir s'est faite avec le temps, soit parce que ces données sont laissées à l'appréciation du cuisinier, entre bon sens et inventivité. Le temps de la recette s'efface au profit du temps légendaire, héroïque, qui donne à la cuisine ses lettres de noblesse comme art culinaire - tekhnê et pas simplement praxis. Sur l'axe géographique, spatial, territorial, les titres font référence à l'origine de la cuisine, à l'empreinte locale, culturelle, avant que l'invention récente du mot "terroir» ne vienne développer et entretenir toute la mythologie du lieu, de la culture et des savoirs attachés à un climat local. À cet égard, Le Trésor gastronomique de France, de Curnonsky et de Croze (1933), contribuera à cultiver l'estime et la nostalgie de terroirs, en fait nourris par l'inspiration des gastronomes, pour comprendre et reconnaittre en quoi la France est bien le pays «où l'on mange et l'on boit le mieux»! À travers les titres, on s'emploie donc à célébrer les régions, les produits, les savoirs (recettes). Et s'il faut cultiver la mythologie d'un Chef, on ira chercher des sources dans sa culture, son enracinement (isotopie du lieu), ou dans un style propre à une époque, voire à un changement d'époque (dimension temporelle)... à moins que le génie du Chef ne soit au croisement de ces deux dimensions à la fois concrètes et symboliques.

Mais, à regarder de plus près, l'effet de catalogue suggéré par le titre ne résiste pas à la lecture. Il suffit d'un regard sur la table des matières, une plongée, même rapide, dans les propos de quelque chapitre pour que l'annonce du titre soit compromise par la liberté de l'auteur avec le genre.

Jusqu'au XIXe siècle, dans la littérature culinaire ou la cuisine dite préscientifique, l'esprit l'emporte sur la lettre. Le discours de l'épicurien, du gastronome, de l'homme lettré a besoin d'espace et de liberté pour trouver la distance avec son objet: tantôt réflexive, sur le mode de l'introspection, du rapport intime avec la sensation alimentaire; tantôt réfléchie, à propos de la sagesse du goût, son humanité, son rapport vivant et profond à la vie en société.

Bien souvent, l'imagination gastronomique s'évade du titre culinaire. Les plaisirs de bouche sont exhaussés par les saveurs de la langue et du discours. En remontant aux références les plus anciennes, les recettes assez imprécises qui nous sont parvenues d'Athénée - compilateur égyptien du III siècle ap. J.-C. -, et rassemblées dans Les Deipnosophistes (Le Diner des savants), prennent moins d'importance que les citations foisonnantes, tant d'écrivains que de cuisiniers. Dès l'origine, donc, la cuisine en appelle à la saveur et à la sagesse, au savoir (qui ont une étymologie commune: sapere). Si le titre donne une coloration savante ou scientifique, on trouve en fait, à l'intérieur des ouvrages, moins un discours technique sur les recettes elles-mêmes qu'une digression littéraire ou philosophique sur la table et la gastronomie. 
Ce trait, déjà marquant chez Athénée, est particulièrement sensible dans les grands ouvrages de l'histoire culinaire, comme De Honesta Voluptate et valetudine (De l'Honneste Volupté), paru en 1473, sous la plume de Platine de Crémone, pseudonyme de Bartolomeo Sacchi. À la Renaissance, cet ouvrage servira de manuel de savoir-vivre et d'art de vivre dans l'Europe humaniste, en cultivant un nouveau regard sur la gastronomie et en marquant l'entrée dans la modernité culinaire. Toujours dans le registre des voluptés du discours, une figure comme Grimod de la Reynière invente le style moderne de la critique gastronomique. Interdit de critique théâtrale, il a l'idée de publier un périodique, qui connaît un énorme succès, comportant un itinéraire des cafés, des restaurants et des boutiques (l'Almanach des gourmands, première édition: 1803). En 1808, il publie le Manuel des amphitryons conçu pour enseigner l'art de recevoir. Il crée également les "jurys dégustateurs", soit autant de manifestations du regard moderne sur la gastronomie, qui se déplace de la technicité du Chef à la compétence du gourmet.

Un enjouement que l'on retrouve chez BrillatSavarin et sa fameuse Physiologie du goût (1825), dont le titre agit, là encore, en trompe-l'œil par rapport au vrai centre d'intérêt de l'auteur, moins homme de science qu'homme de lettres: «De bout en bout, le livre du proprement humain, car c'est le désir (en ce qu'il se parle) qui distingue l'homme» (Barthes, 1975: 9). Or, l'ouvrage, dont le titre complet est Physiologie du goût, ou Méditations de gastronomie transcendante; ouvrage théorique, historique et à l'ordre du jour, dédié aux gastronomes parisiens, se présente comme une invitation, une initiation aux principes généraux de la "gastronomie», terme de création récente, dérivé du grec.

Les premières Méditations, tout particulièrement, s'inscrivent dans le cadre littéral de la physiologie et de la chimie. Mais, en considérant le plaisir de la table au-delà du plaisir de manger ${ }^{2}$, Brillat-Savarin, comme il l'indique dans sa préface, voit de bonne heure qu'il y a «quelque chose de mieux à faire que des livres de cuisine».
La Physiologie, malgré le titre, se révèle comme une confession, une "confabulation" (entretien familier), une collection de notes personnelles et de «méditations» profondes, qui percent l'intimité du goût, la dévoilent, dans toute son humanité, ce qui est l'essence même de l'ouvrage, sa force et son caractère.

À ce stade, il nous faut donc convenir que le titre, tout en fixant une orientation par rapport à un genre discursif (dictionnaire, manuel, traité, livre, etc.), par rapport à un type de discours (technique, scientifique, littéraire, philosophique) ou par rapport à un axe de valorisation (temporel, spatial, actoriel ou auctoriel - en référence au cuisinier, au Chef), ne peut contenir l'imagination de l'auteur qui, le plus souvent, suit la trajectoire métonymique ou synecdochique du plat ou de la recette jusqu'au contexte social ou symbolique qui déploie la scène gastronomique. Cet espace figuratif trouvera une formidable expansion dans la prolifération des titres d'ouvrages et de revues qui reviennent inlassablement sur cet imaginaire de la cuisine, mais qui cherchent, autant que possible, à marquer la particularité de leur approche. Là où le titre brouillait les pistes, il faut voir aussi comment il peut choisir un angle, délimiter un espace propre à l'intérieur du monde culinaire et contribuer à construire une image plus globale, plus complexe: celle du goût, de l'imaginaire gustatif.

\section{DU TITRE À L'ASSIETTE SÉMANTIQUE DU GOÛT}

Si l'on se reporte au plus ancien livre de cuisine attribué à Apicius ${ }^{3}$, on trouve une collection de recettes classées par ingrédients et par plats, mais les éditions successives, à partir de la fin du XVe siècle, jouent précisément sur le titre pour mettre, tour à tour, l'accent sur l'art (Ars magirica, du grec mageiros, cuisinier), sur l'homme (Apicius culinarius), sur la collection des recettes (De Re Coquinaria libri dicem: Les Dix livres de cuisine) ou sur la cuisine tout court (De Re Coquinaria) - un déplacement significatif des différents modes de représentation du monde culinaire.

Sur quoi, en fait, convient-il d'accommoder le regard: sur la cuisine, versant production, ou sur la gastronomie, versant consommation? Ce 
découpage serait trop simple et dichotomique pour rendre compte des opérations de chiasme: la cuisine anticipe ou préfigure l'art de goûter, de savourer, elle prédispose à un certain mode de réception; la gastronomie démêle, quant à elle, la savante organisation des ingrédients et des saveurs conçue et réalisée par le cuisinier. En outre, l'art relève-t-il de la technique ou de la dégustation, de la création culinaire ou de la récréation gastronomique? Il n'y a pas de coupure entre les deux mondes, mais bien davantage des correspondances d'univers, des liens étroits et métonymiques entre les causes et les effets, la chimie des saveurs et l'alchimie des valeurs incorporées avec la nourriture, partagées à table. Ce que Barthes consigne dans sa «Lecture de Brillat-Savarin", à l'article "Topique», nous pouvons l'appliquer à la multiplicité des approches qui se donnent pour objet la cuisine et la gastronomie:

Autrement dit, le discours est en droit d'attaquer la nourriture sous plusieurs pertinences; c'est, en somme, un fait social total, autour duquel on peut convoquer des métalangages variés: ceux de la physiologie, de la chimie, de la géographie, de l'histoire, de l'économie, de la sociologie et de la politique (nous y ajouterions, aujourd'hui, la symbolique). C'est cet encyclopédisme - cet «humanisme» - que recouvre, pour Brillat-Savarin, le nom de gastronomie. (1975: 32)

À charge, donc, pour les ouvrages et les revues d'attaquer sous le meilleur angle cet univers encyclopédique qui, au-delà des aliments et des recettes, recouvre tant de réalités et nourrit tant de symboles. Sous l'angle premier du titre, les références à l'histoire culinaire nous invitent déjà à la prudence, car, sous les termes génériques en tête d'ouvrage, se cache puis se détache, comme on l'a observé, la libre inspiration des auteurs qui conjuguent recettes de cuisine et cuisine du sens, fussent-ils cuisiniers ou critiques gastronomiques. En regardant du côté non seulement des ouvrages mais aussi des revues culinaires, qui se comptent par centaines, on peut voir si la fonction des titres a évolué avec celle de l'offre, tant la littérature gastronomique, au sens large, étend son territoire.
Sachant la profusion des angles d'attaque sur lesquels il peut jouer, il est évident que le titre doit garder:

- une valeur d'accroche pour le lecteur, au sens phatique et poétique, qu'il s'agisse d'un ouvrage original ou d'une revue soucieuse d'exprimer, dès ce niveau d'accès, son esprit de création;

- une valeur différentielle par rapport à l'inflation des titres sur le marché de l'édition - ce en quoi le titre permet de se positionner, de marquer non seulement une différence, mais peut-être aussi un avantage concurrentiel sur les autres, par le thème, l'angle, la thématique, la nature du propos;

- une valeur d'identité, qui affirme un caractère, un style, une image, dans la façon d'aborder cet encyclopédisme de la cuisine et de faire des choix stratégiques. Il faudrait, à cet égard, prendre en compte les éléments graphiques et visuels qui participent de cette identité du titre, de son originalité et de sa reconnaissance par les lecteurs, les publics. Nous ne pourrons le faire, dans les limites de cet article, mais c'est une dimension à retenir.

Ce sont des logiques de création et de production tellement diversifiées, qu'il faut, selon nous, chercher un principe organisateur dans la profusion des titres, chercher de l'unité là où pourraient n'apparaître que la dispersion du champ alimentaire, l'émiettement et la confusion entre tous les discours possibles à tenir, finalement déconnectés les uns des autres. À l'inverse, cette profusion même des titres ne permet-elle pas de dresser, sinon une cartographie un peu longue à justifier, du moins un parcours figuratif de ce monde alimentaire, culinaire, selon ses principaux niveaux d'expression et d'extension? C'est l'hypothèse que nous ferons, à partir du concept de figurativité du goût (Boutaud, 2005), plutôt que figurativité de la nourriture ou de l'alimentation, qui subsument et débordent le culinaire et le gastronomique. Ce concept a été développé dans nos recherches antérieures sur la sémiotique alimentaire, mais nous devons l'appliquer, mutatis mutandis, pour 
organiser la profusion des titres selon un principe unificateur.

En contrepoint de Leroi-Gourhan qui parle, pour la gastronomie, d' "esthétique sans langage» (1965: 113), Floch estime, au contraire, que:

La cuisine est bien un langage: elle n'est pas un jeu gratuit d'odeurs, de goûts et de consistances. Et de plus, comme tous les autres langages, la cuisine peut devenir l'objet d'un métadiscours, portant ou sur son instance de production (l'art culinaire) ou sur son instance de saisie (la gastronomie).

Notons qu'il est inutile, dans le cadre de cette approche, de s'attarder sur l'aspect figuratif des plats, des compositions culinaires, des ordonnancements de table, qui constituent autant de «langages» pour la préfiguration et la reconnaissance du goût. Il sera plutôt question, maintenant, de voir en quoi les titres contribuent à l'extension figurative du goût, au sens alimentaire, précisément par la mise en tension entre ce qui serait de l'ordre de la production, les arts culinaires, et de la reconnaissance, la gastronomie, si l'on s'accorde avec Floch sur cette distribution. Du côté des arts culinaires, on compte gestes, techniques, produits et recettes, mais aussi chefs, cuisines et cuisiniers de toutes sortes. Du côté de la gastronomie, on retient le plaisir de se mettre à table, de savourer, de partager, mais aussi le décor et la mise en scène alimentaire, avec sa dramaturgie et tous ses codes de figuration, respectés ou détournés.

Les aliments, la cuisine, la gastronomie constituent tout un univers qui s'organise autour du mangeur, de l'acte alimentaire; toutes ces données, à la fois pratiques et symboliques, se condensent, en fait, dans un terme syncrétique: le goût ou, d'un point de vue discursif, l'image du goût. De saveur en valeur, de sensation figurée, exprimée, en représentation et mise en scène (en sèmes), il parcourt bien des étages, bien des étapes de notre sensibilité alimentaire, à travers des niveaux figuratifs qui en déploient le discours, l'image, la communication. Il est possible, dès lors, de situer les titres des ouvrages et des revues par rapport à ces niveaux d'expression discursive, figurative (objets, images, espaces, actions mises en scène, formes de vie), autrement dit par rapport à tous les niveaux de présentation et de médiation du goût.

Appliqué à ce domaine, tout particulièrement dans le registre des compétences, pour ne pas dire des performances liées à la cuisine et à la table à l'intérieur du vaste monde alimentaire, le concept de figurativité peut aussi se concevoir à d'autres niveaux expressifs ${ }^{4}$ par rapport à ceux déjà entrevus. Dans notre visée, ils correspondent à autant de niveaux de distribution des titres:

- Niveau substantiel: le titre marque la valorisation du contact esthésique (sensations), synesthésique (correspondances sensorielles) avec les aliments, au plus près de la perception sensorielle (mots clés: saveurs, sensations gourmandes, goûter, déguster).

- Niveau référentiel et sectoriel: le titre renvoie explicitement à un produit, un type d'aliments, un secteur, qu'il s'agisse d'un discours pour amateurs éclairés ou pour professionnels; ce qui nous écarte, alors, de l'isotopie du goût, en termes culinaires, gastronomiques, au profit de considérations plus techniques, marchandes, pour les métiers concernés (mots clés: aliment, produit, propriétés, qualité, marché).

- Niveau thématique: le titre s'attache à un thème ou à un domaine, la frontière n'étant pas toujours facile à établir si l'on considère, par exemple, le discours sur le bio (principe de culture, gamme de produits, culture alimentaire tout court). À ce niveau, les revues et les ouvrages saisissent des thèmes à la fois émergents et sans cesse «revisités»: santé, nature, diététique, vitalité, exotisme, etc.

- Niveau technique et opératoire: ce niveau concerne, avant tout, le besoin et l'utilité, mais aussi le désir et le plaisir d'aborder la cuisine comme pratique, comme technique, dans une tension bénéfique, pour la création des titres, entre cuisine pratique et art de cuisiner. Cela se traduit, dans la forme contractuelle du titre, par un appel au geste culinaire, à l'habileté du cuisinier ou de la ménagère. Ces activités pratiques sont gouvernées 
par la nécessité (faire court, simple, facile, etc.) ou par l'envie de se dépasser (cuisine créative, astuces, imitation), ce qui n'est pas toujours contradictoire, loin de là : «cuisine facile et créative», par exemple, constitue un lieu commun culinaire (mots clés: cuisine, cuisiner, cuisinier). En se déplaçant du geste pratique au style, on marque aussi un déplacement de la technique à l'identité culinaire.

- Niveau identitaire: le titre met en avant la figure d'un Chef, avec son caractère, sa personnalité, sa cuisine, son univers. C'est la figure d'autorité, dominante à tous les sens du terme, qui va servir de guide dans la construction d'une forme de vie alimentaire. L'identité culinaire s'exprime aussi, dans le titre, par référence à un pays, un terroir, un territoire, entre discours pratique (produits, recettes) et imaginaire alimentaire. Cuisine d'ici et d'ailleurs, du présent et du passé, cuisine savante et cuisine populaire, paysanne ou "bourgeoise», des «profondeurs» (Revel), voilà autant de choix possibles pour inventer un titre, quand ce n'est pas le Chef lui-même qui le porte.

- Niveau situationnel et interactionnel: de la cuisine à la table, de la préparation à la dégustation, les titres des ouvrages et des revues recomposent l'espace figuratif de la scène alimentaire: espace d'exposition, qui préfigure le repas, par exemple; espace de figuration pour le mangeur, les convives, plongés dans des situations extrêmement différentes, en privé ou en public. C'est la mise en scène des produits, des acteurs, et les scènes de tables, scènes de vie, qui exploitent tout le champ métonymique du goût: saveur des aliments et saveur du partage, du lien. Sur toile de fond sociale, ressortent les usages, les codes, les bonnes manières, comme tous les jeux autour de la table (mots clés: table, goût, histoire, manières, etc).

Perçue dans l'articulation de ses niveaux figuratifs, à travers discours, acteurs, lieux, situation, l'image $d u$ goût offre donc, comme nous le disions en rappelant la «Lecture de Brillat-Savarin» par Barthes, de multiples angles d'attaque pour parler cuisine et gastronomie et concevoir des titres dignes d'intérêt.

Avec les ressources du réseau Internet et des supports de communication multimédias, dont l'explosion manifeste une inventivité sans pareille, il est possible que les titres trouvent un terrain de liberté et de créativité, offrant un espace toujours plus grand à notre imaginaire alimentaire. C'est ce qu'il faut essayer d'entrevoir maintenant.

\section{ÉTHIQUE ET POÉT(H)IQUE DES BLOGUES}

Suivons bien la courbe des productions culinaires: une histoire fourmillant de titres qui mettent en avant la cuisine, l'art de cuisiner, mais ne donnent qu'un rôle secondaire aux recettes elles-mêmes. Se lit un enrichissement continu du discours, autour d'une figure centrale, le goût, avec la multiplication des angles d'entrée, d'attaque, traduite dans les titres mêmes. À travers cela, s'exprime une sensibilité gastronomique qui se développe à tous les niveaux de représentation (les produits, les techniques, les lieux, les acteurs), mais sous la figure dominante du Chef qui montre la voie, du geste qui requiert de la maîtrise, du bon usage qui respecte les codes, les nourrit même, en évitant précisément les fautes de goût.

Avec l'avènement d'Internet et des blogues culinaires, la balle semble passer dans l'autre camp, le mangeur reprend la main, si l'on peut dire. L'imaginaire de la cuisine, d'abord arrimé au discours des sciences, puis séduit par le discours des arts, semble aujourd'hui donner la priorité à l'expérience du sujet et à sa propre mise en scène dans l'espace culinaire. On se déplace ainsi du discours expert du Chef ou du critique gastronomique, à la performance culturelle de celui ou celle qui fait la cuisine en cultivant, de façon déculpabilisée, amusée ou très impliquée, un ethos et une esthétique du faire culinaire et gastronomique. Cela explique le rôle péritextuel des légendes qui accompagnent les titres des blogues et des sites, en pointant cette direction expérientielle dans le rapport à la cuisine. 
De la compétence culturelle à reproduire le bon geste, à imiter le Chef, à se conformer à l'étiquette du beau et du bien, on glisse, imperceptiblement, à la performance du mangeur ordinaire, de l'amateur de table et de cuisine, sans autre prétention que se faire plaisir et, pourquoi pas, faire partager ce plaisir aux autres. En première approche ou par choix, il n'y a pas là de quoi trouver un éditeur, ni donner matière à tout un livre, mais suffisamment pour s'exprimer en toute fraîcheur, voire candeur. S'élabore ainsi un espace personnel, de liberté, de créativité culinaire sans le surmoi ou le surplomb de quelque figure autorisée pour parler cuisine, la réaliser et la faire connaître. Les titres des blogues participent de cette expression libérée, détendue, jouant des marques énonciatives pour créer un contact direct et franc.

Prenons quelques exemples 5 . Alhya, avec A Turtle in a Kitchen (http://aturtleinakitchen.blogspot.com), brosse d'emblée son univers:

Ce n'est pas vraiment un recueil de recettes, égrainant ingrédients et méthodes. C'est l'histoire d'une suite de découvertes, d'amusements, de challenges, tous enrichissants, prenant corps derrière mes fourneaux et se nourrissant de la vie, en général. 6

Chez Cléa aussi (www.cleacuisine.fr/portrait), il est question avant tout de s'amuser:

J'ai 27 ans et je cuisine pendant mon temps libre, en dehors

de mon travail (qui n'a, lui, rien à voir avec la cuisine, merci bien!). Je suis donc une amatrice, passionnée, et mon plus grand plaisir est de créer sans cesse de nouvelles recettes pour amuser mes papilles et celles de mes proches.

On pourrait multiplier, ainsi, les témoignages de tous ces esprits créatifs qui prennent en main leur destin culinaire et livrent, en toute franchise, leur univers personnel, intime, fait de goûts et d'envies, de passions et de rencontres, de recherches et de trouvailles - expériences que chacun d'entre nous est en mesure de ressentir et de vivre au quotidien.

Là encore, la cuisine peut se prêter au discours catachrétique, quand l'humeur et l'imagination s'émancipent du propos culinaire, s'éloignent des fourneaux et travaillent, avant tout, à créer un univers. Mais cuisine et recettes sont toujours le point de départ de cet imaginaire fertile qui cultive moins les connaissances que les impressions, moins la technique que les instants et les émotions.

Les titres prennent souvent une forme ludique:

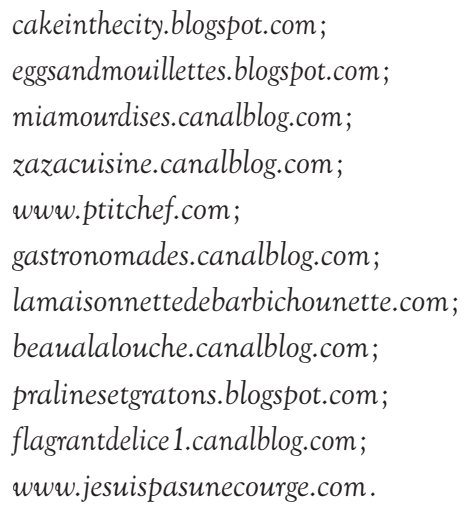

L'empathie et la connivence s'affichent dans les prénoms:

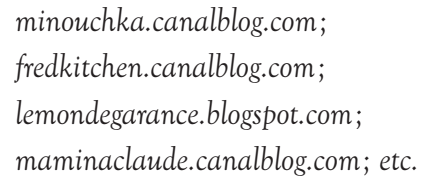

La forme même des adresses, sur les sites, se prête à ces manipulations ludiques, poétiques, en raison de l'obligation de contracter les termes. Ces adresses deviennent des sortes de witz, de mots-valises, de calembours, exploitant, comme on peut le voir dans les titres déjà mentionnés, les ressources du sémantique, du graphique et du phonétique (par exemple: lamaisonnettedebarbichounette.com).

À travers Internet et les blogues, on voit donc se dessiner, plus que jamais, un ethos culinaire préfiguré par le titre. Il s'agit d'un ethos à deux niveaux: à travers l'affirmation libre d'une forme de vie culinaire, d'un style de vie épanoui, possible à reconstruire

[...] entre des forces «centrifuges» - les pulsions, les passions,

l'imaginaire et les interactions de l'ego -, et des forces

"centripètes» - civilité, normalisation des images corporelles, 
contraintes diététiques, économiques ou commerciales, cadres sociaux d'appartenance, etc. (Corbeau, 1996: 182)

De forme constructiviste, l'ethos laisse ainsi place à l'imaginaire du mangeur, du cuisinier, qui «bricole» son rapport à la nourriture, moins par désir de maîtrise que par réveil du désir lui-même.

Par sa forme énonciative, connivente, ludique, inventive et décalée, le titre du blogue invite donc à partager un ethos culinaire, un ethos participatif, lié très précisément à la forme interactive du blogue, qui redouble la forme de sociabilité déjà entrevue dans le sens premier de l'ethos. L'invitation à découvrir le blogue, dans le titre, est plus encore invitation à partager un univers culinaire

[...] non pas seulement comme une organisation

d'informations et d'instructions mais comme l'espace où

l'énonciateur et son énoncé se mettent en scène, invitant ainsi l'usager, alors co-énonciateur, à adhérer à la manière de communiquer. C'est ainsi que chaque annonceur, chaque éditeur de presse en ligne, entre autres, cherchent, à travers les interfaces des "pages-écran" proposées sur leur site, à mettre en scène leur ethos, un Faire social, une représentation morale et imaginaire qu'ils nous invitent à adopter.

(Pignier, 2008: 32)

La remarque prévaut pour tous les espaces numériques qui ajoutent le bricolage participatif du support de communication au bricolage du discours dans l'univers alimentaire. Ce qui transparaît désormais, dans la liberté et l'inventivité des titres sur Internet, avec tous les signes énonciatifs de la connivence, de la proximité, de la détente, c'est bien l'expression enjouée d'un ethos, en termes de sociabilité (Corbeau) et comme forme de vie partagée (Pignier).

L'éthique de la relation vient donc redoubler la poétique du titre. Avec le blogue et Internet, il témoigne d'un esprit plutôt léger et badin, là où le livre privilégiait la science et le discours hautain du Chef, du critique, de l'expert. On peut supposer que la production livresque cherchera, de plus en plus, à s'inspirer de cette créativité dans le titre, mais sans espoir de concurrencer ce qui est laissé à l'initiative individuelle, aux audaces personnelles, dans la production éditoriale des blogues culinaires.

Contre les incantations et l'anathème sur la malbouffe, sur la mort du goût et l'érosion de l'imaginaire de table, la vitalité du titre culinaire semble témoigner, au contraire, d'un regain d'intérêt, pour tout ce qui touche à la nourriture, au désir de bien manger, au plaisir de table, s'il fallait rester sur les pas d'un Brillat-Savarin. Mais l'humanisme du goût a longtemps été porté, dans l'histoire culinaire, par une intellectualisation du goût, par une professionnalisation du geste, une technicité de la pratique et une morale du discours, autant de dimensions suggérées dans les titres mêmes des ouvrages érudits ou savants, à prétention scientifique ou littéraire, voire artistique. L'évolution du discours et des pratiques profite à une démocratisation du faire culinaire, à une esthétisation du quotidien, articulant, sur le mode de la performance ordinaire, auto-réalisatrice, ce qui pouvait se comprendre auparavant comme une compétence technique, avide de conseils et de leçons. En jouant plutôt la proximité, la familiarité, les titres, notamment sur Internet, marquent un changement de ton dans l'approche de la cuisine familiale. La vie se veut désormais portée par le réenchantement de l'expérience. L'émotion culinaire, gastronomique, participe de ce besoin, de cette recherche, du versant utilitaire ou pratique des gestes au quotidien, au raffinement esthétique, mais créatif, ludique. La polychrésie ${ }^{7}$ (grec: krèsthaï, «user de») du mode alimentaire se soutient de la polyphonie des discours sur le monde culinaire, faisant usage du titre comme d'une bannière haute en couleurs et en saveurs expressives. Derrière les mots, des modes de vie alimentaires, des espaces de pratiques, qui, pour paraphraser Revel (1979), font place au cuisinier pensant qui crée, au cordon bleu qui suit la recette, au gourmand ou au gourmet qui savourent, si ce n'est au mangeur ordinaire que nous sommes au quotidien, pour peu que le titre nous mette en appétit. 


\section{NOTES}

1. Selon le Littré, le traité est un "ouvrage où l'on traite de quelque art, de quelque science, de quelque manière particulière» (1978, tome 4 : 6423).

2. «Le plaisir de manger est la sensation actuelle et directe d'un besoin qui se satisfait. Le plaisir de la table est la sensation réfléchie qui naît des diverses circonstances de faits, de lieux, de choses et de personnages qui accompagnent le repas " (Brillat-Savarin [Méditation XIV, «Du plaisir de la table»], 1975 : 111).

3. Apicius recouvrait en fait plusieurs personnes (Ier siècle av. J.-C. - II ${ }^{\mathrm{e}}$ siècle ap. J.-C.), dont la plus célèbre, Marcus Gavius Apicius (né en 25 av. J.-C.).

4. On pourrait également parler de plans figuratifs. Mais le concept de figurativité engage à passer d'un niveau à l'autre dans le déploiement du goût, de l'expression de la sensation la plus directe, l'image sensorielle, à tous les niveaux de mise en scène alimentaire. Ils relèvent des formes de médiation et de figuration des pratiques de cuisine, de table, possibles à vivre comme des performances culturelles. Nous renvoyons, là encore immodestement, à notre article: «Du goût à l'image gustative. Un déploiement figuratif " (2004).

5. Je tiens à remercier Alexandra Beauvais (doctorante à l'Université de Bordeaux - Laboratoire Imagines) qui m’a aidé dans le repérage des constantes discursives sur Internet, avec une liste de blogues culinaires impossible à reproduire dans les limites de cet article. Nous renvoyons au no 1 de Cuisine P@ssion (février-mars 2008) qui propose un inventaire de 29 blogues particulièrement originaux. Pour une information beaucoup plus élargie, voir The Food Section. All the news that's fit to eat. En ligne: www.thefoodsection.com/foodsection/links.html (page consultée le 15 octobre 2008).

6. Cet extrait n'était pas accessible à l'automne 2008.

7. Néologisme de Y. Jeanneret (2008)

\section{RÉFÉRENCES BIBLIOGRAPHIQUES}

BARTHES, R. [1975]: "Lecture de Brillat-Savarin", dans A. BrillatSavarin, 7-33.

BouTAUD, J.-J. [2004] : «Du goût à l'image gustative. Un déploiement figuratif ", Voir barré, n 28-29 (II. Le goût et l'odorat), novembre, 24-33;

[2005]: Le Sens gourmand. De la commensalité, du goût, des aliments, Paris, Jean-Paul Rocher.

Brillat-SaVarin, A. [(1825) 1975]: Physiologie du goût, Paris, Hermann, coll. «Savoir".

CORBEAU, J.-P. [1996] : «De la présentation dramatisée des aliments à la représentation de leurs consommateurs ", dans I. Giachetti (dir.), Identités des mangeurs. Images des aliments, Paris, Polytechnica, 175-198. Dumas, A. [1873]: Grand Dictionnaire de cuisine, Paris, Alphonse Lemerre.

FLOCH, J.-M. [1995]: Identités visuelles, Paris, PUF.

FONTANILLE, J. [2007]: "Textes, objets, situations et formes de vie. Les niveaux de pertinence de la sémiotique des cultures", dans J.A. Aldama, D. Bertrand, M. Costantini et S. Dambrine (dir.), La Transversalité du sens, Paris, PUV, coll. "Essais et savoirs".

JeANNERET, Y. [2008] : Penser la trivialité. La vie triviale des êtres culturels, Paris, Hermès Lavoisier.

Leroi-Gourhan, A. [1965]: Le Geste et la Parole: la mémoire et les rythmes, Paris, Albin Michel.

Pignier, N. [2008] : Social Experience du Web Design, Paris, Hermès Lavoisier.

REVEL, J.-F. [1979]: Un festin en paroles. Histoire de la sensibilité gastronomique, de l'Antiquité à nos jours, Paris, Jean-Jacques Pauvert. 